\title{
(2) OPEN ACCESS \\ Ultrasonographic needle tip tracking for in-plane infraclavicular brachialis plexus blocks: a randomized controlled volunteer study
}

\author{
Trine Kåsine (1) , 1,2 Luis Romundstad, ${ }^{3}$ L A Rosseland, ${ }^{1,2}$ Morten Wang Fagerland, ${ }^{4}$ \\ Paul Kessler, ${ }^{5}$ Ivar Nagelgaard Omenås, ${ }^{3}$ Anne Holmberg, ${ }_{1}^{3}$ Axel R Sauter ${ }^{1,3,6}$
}

\begin{abstract}
${ }^{1}$ Division of Emergencies and Critical Care, Department of Research and Development, Oslo University Hospital, Oslo, Norway

${ }^{2}$ Institute of Clinical Medicine, Faculty of Medicine, University of Oslo, Oslo, Norway

${ }^{3}$ Division of Emergencies and Critical Care, Department of

Anesthesiology, Oslo University Hospital, Oslo, Norway

${ }^{4}$ Oslo Centre for Biostatics and Epidemiology (OCBE) Research Support Services, Oslo University Hospital, Oslo, Norway

${ }^{5}$ Department of Anesthesiology, Intensive Care and Pain Medicine, Orthopedic University Hospital Friedrichsheim, Frankfurt am Main, Hessen, Germany

${ }^{6}$ Department of Anesthesia and Pain Medicine, Inselspital Bern University Hospital, Bern, Switzerland
\end{abstract}

Correspondence to Dr Trine Kåsine, Anesthesiology, Oslo University Hospital, 0424 Oslo, Norway; trikaa@ous-hf.no

Received 6 February 2020 Revised 12 May 2020 Accepted 18 May 2020 Published Online First 14 June 2020

\begin{abstract}
Background Onvision is a new technology for needle tip detection and tracking in ultrasound-guided regional anesthesia. The system consists of a piezoelectric sensor close to the needle tip and an electronic console integrated in the ultrasound system. The needle tip is visualized by a green circle on the ultrasound screen. The aim of the study was to investigate the effect of the new needle tip tracking technology on in-plane infraclavicular plexus blocks.

Methods The study was a randomized, controlled, observer blinded cross-over trial in 26 healthy volunteers. Two specialists in anesthesiology performed an ultrasound-guided infraclavicular lateral sagittal brachial plexus block with and without needle tip tracking. Primary outcome was procedure time, measured from insertion of the needle until local anesthesia injection was completed. Secondary outcome measures included the number of hand movements and path lengths (assessed by hand motion analysis), block success rate, onset time and duration, discomfort experienced by the volunteers, and the anesthesiologists' confidence as to whether their block would be successful or not.

Results Mean (SD) procedure time was 183.0 (56.1) s with and 206.8 (56.2) s without needle tip tracking $(p=0.16)$. There were no significant differences in any of the secondary outcomes. Two volunteers' experienced Horner syndrome after blocks without needle tip tracking. No other adverse events occurred during the study.

Conclusion Our study on needle tip tracking for infraclavicular blocks did not reveal any significant differences between active needle tip tracking and the control procedures, neither for primary outcome nor secondary outcome measurements.

Trial registration number NCT03631914
\end{abstract}

\section{INTRODUCTION}

In spite of the use of ultrasound guidance, peripheral nerve blocks still have risks for block failures and complications. ${ }^{1}$ Failure to identify the correct needle tip position can explain both complications and block failures. The use of a reliable needle tracking system can potentially improve feasibility, precision, effectiveness, and safety of nerve block techniques. $^{2}$

The Onvision needle tip tracking technology (B. Braun Melsungen AG, Melsungen, Germany and Philips Medical Systems International B.V.,
Eindhoven, the Netherlands) is a new needle tip tracking technology customized for use in regional anesthesia. Our research group has previously tested this technology in a randomized controlled trial with a porcine phantom model. ${ }^{3}$ The use of the needle tip tracking technology significantly reduced the procedure time and the number of hand movements for out-of-plane procedures. In a consecutive human volunteer study on out-of-plane lumbar plexus blocks, we found a significantly reduced number of hand movements and path length indicating a better needle control when needle tip tracking was used. ${ }^{4}$

The effect of needle tip tracking used for an ultrasound-guided in-plane block procedure has not yet been investigated in a randomized trial on human subjects.

The aim of our study was to investigate the effect of needle tip tracking on the performance of ultrasound-guided infraclavicular brachial plexus blocks in healthy volunteers. We hypothesized that needle tip tracking could reduce the procedure time for infraclavicular brachial plexus blocks performed with an in-plane needle approach in healthy volunteers.

\section{METHODS}

This randomized controlled cross-over human volunteer trial was conducted between September 3, 2018 and September 28, 2018, with enrollment from August 20, 2018. A monitor from the Clinical Trial Unit at Oslo University Hospital oversaw the conduct of the trial. Good clinical practice was followed throughout the study. All volunteers included gave informed written consent.

\section{Volunteers}

Twenty-six healthy volunteers were recruited by bulletins in the hospital and university and by social media. Volunteers aged 20-65 years with American Society of Anesthesiologists physical status classification 1 or 2 , body mass index $18-35 \mathrm{~kg} / \mathrm{m}^{2}$, and weight $<95 \mathrm{~kg}$ were eligible for participation. They had to be able to understand and speak Norwegian. Any kind of neurological disease, nerve or vascular impairment, known coagulopathy, pregnancy, allergies to amide local anesthetic agents, or skin disease affecting the area of examination were exclusion criteria.

\section{Needle tip tracking}

The needle tip tracking technology consists of a Stimuplex Onvision needle (B. Braun Melsungen 


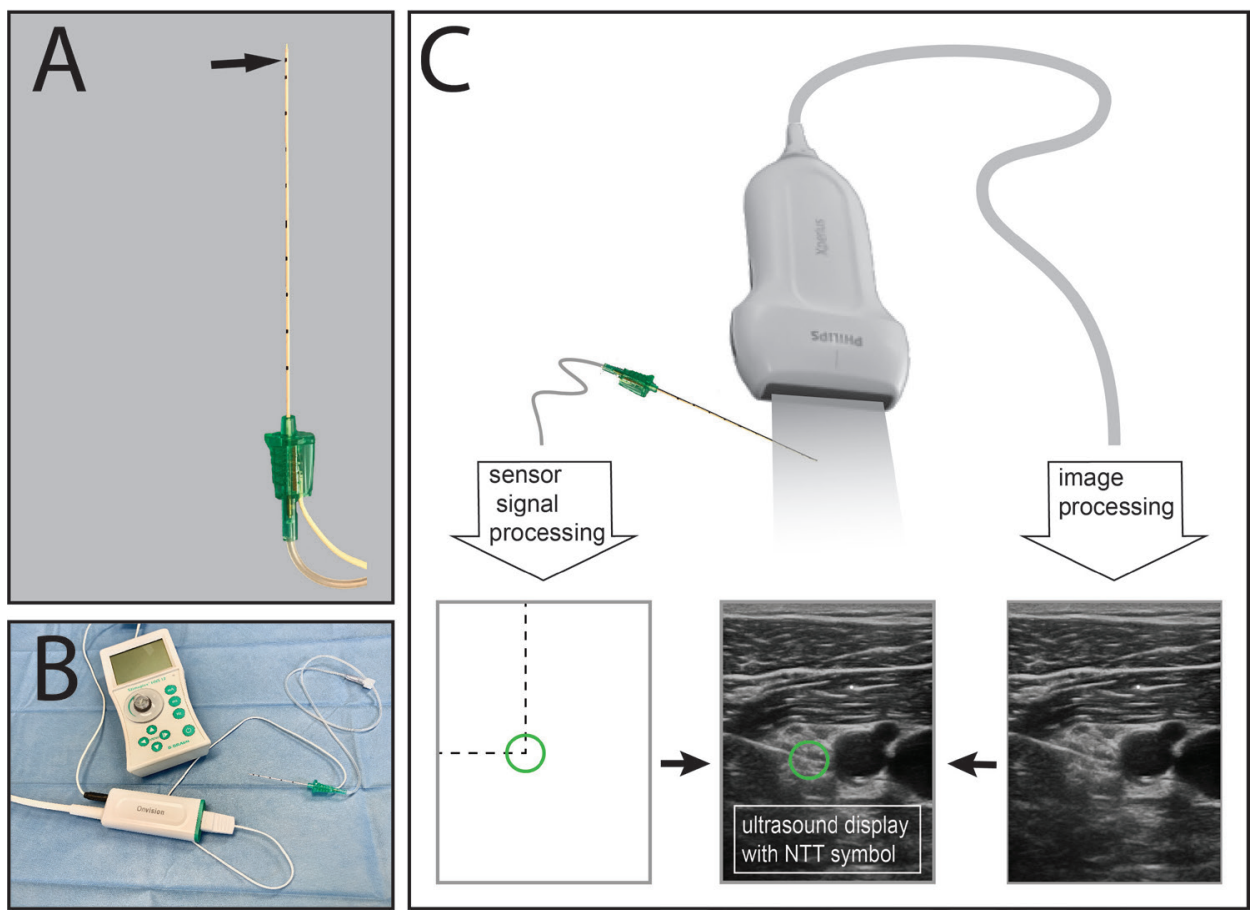

Figure 1 The Onvision needle tip tracking (NTT) technology. (A) A piezoelectric sensor is wrapped around the needle close to the needle tip. The arrow indicates the position of the sensor. The needle has no echogenic texture. (B) The design of the needle is comparable to a standard nerve block cannula with one tube for fluid injections and one electrical wire for signal transmission from the sensor to the connector. The same wire is used for electrical nerve stimulation while connecting the nerve stimulator to the connector. (C) The piezoelectric sensor on the needle collects the ultrasound waves which are sent out by the transducer. A signal processing unit calculates and projects the position of the sensor on the two-dimensional ultrasound image. The needle tip is visualized by a green circle overlay on the ultrasound image.

AG) with an integrated piezoelectric sensor close to the needle tip and an Xperius ultrasound system (Philips Medical Systems International B.V. and B. Braun Melsungen AG), ${ }^{34}$ with an electronic console for computerized signal processing. The piezoelectric sensor on the needle collects the ultrasound waves which are sent out by the transducer. A signal processing unit calculates and projects the position of the sensor on the two-dimensional ultrasound image. The needle tip is visualized by a green circle on the ultrasound image. When the needle is located in the ultrasound beam, a small green circle will be visible on the ultrasound screen. This small green circle represents the position of the piezoelectric sensor on the needle tip (figure 1). For in-plane procedures, the needle tip is located on the circle line, opposite to the side of needle insertion. Even though the needle tip is not in the ultrasound plane, the needle tip tracking system can capture a faint signal. The needle tip is then indicated by a red and blue circle of increasing diameter (dependent on the distance from the sensor in accordance to the ultrasound plane) on the ultrasound screen (figure 2).

\section{Ultrasound-guided infraclavicular plexus block}

Two senior anesthesiologists, with profound experience in ultrasound-guided peripheral nerve block techniques, performed an ultrasound-guided in-plane infraclavicular lateral sagittal brachial plexus block. ${ }^{5}$ Both anesthesiologists had practiced the use of the needle tip tracking technology during a 1-week period on Thiel cadavers, muscle tissue and gelatin phantom models.

The volunteers did not receive sedation or analgesia before or during the procedure. An intravenous cannulation was performed as a safety precaution. Standard monitoring included pulse oximetry, non-invasive blood pressure measurement and ECG. The participants were lying supine on the operating table with the arm to be anaesthetized adducted. Block procedures were performed on the non-dominant side.

An Xperius ultrasound system (Philips Ultrasound, Bothell, Washington, USA) with a linear ultrasound transducer with frequency range from $4 \mathrm{MHz}$ to $12 \mathrm{MHz}$ and a $100 \mathrm{~mm}$ Stimuplex Onvision needle (B. Braun Melsungen AG) with a $30^{\circ}$ bevel was used for infraclavicular blocks. The anesthesiologists performed a prescan to get an overview of the anatomy. Prior to the block needle insertion, the local anesthetic was injected subcutaneously $(2-3 \mathrm{~mL} \quad 20 \mathrm{mg} / \mathrm{mL}$ lidocaine with $5 \mu \mathrm{g} / \mathrm{mL}$ epinephrine). The block needle was advanced in-plane with

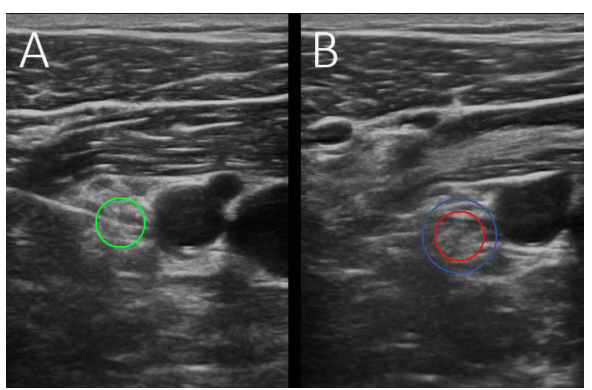

Figure 2 (A) A circle with green color represents the position of the needle tip on the ultrasound screen when the needle is in the ultrasound plane. For in-plane procedures, the needle tip is located on the circle line, opposite to the side of needle insertion. (B) When the needle tip is outside of the ultrasound plane, the system can still pick up a faint signal. The needle tip is then indicated by a red and a blue circle of increasing diameter on the ultrasound screen (dependent on the distance from the piezoelectric sensor on the needle in accordance to the ultrasound plane). 
the ultrasound beam and placed in a central position between the cords of the brachial plexus close to the axillary artery. Depending on the visualized spread of the local anesthetic, the needle was redirected to achieve an adequate local anesthetic around the different nerve cords. An electrical nerve stimulator (Stimuplex HNS 11; B. Braun Melsungen AG) was used with a current setting of $0.5 \mathrm{~mA}, 0.1 \mathrm{~ms}$ impulse duration, and $2 \mathrm{~Hz}$ frequency to minimize the risk of intraneural injection ("dual guidance”). If motor response was elicited with $0.5 \mathrm{~mA}$ or below, the needle was withdrawn until no response was seen at this current intensity. Independently of the weight of the volunteers, we injected $20 \mathrm{~mL}$ lidocaine $20 \mathrm{mg} / \mathrm{mL}$ with epinephrine $5 \mu \mathrm{g} /$ $\mathrm{mL}$.

\section{Randomization and blinding}

This study was a randomized controlled A-B/B-A cross-over trial. Each volunteer received one block with needle tip tracking and one without needle tip tracking. There was a 3-week washout period between blocks. Volunteers were allocated into groups of equal size using a list of random numbers, according to the Moses-Oakford algorithm. ${ }^{6}$ Randomization was done by a person not participating in the study. The observers doing the assessments were blinded for the group allocation. The observers recording data during the block procedure could not see the ultrasound screen and were blinded to whether or not needle tip tracking technology was being used. Also, the volunteers were blinded for the use of needle tip tracking.

\section{Outcome and assessments}

The primary outcome measure was procedure time, defined as the time from insertion of the block needle (skin puncture) until local anesthesia injection was completed. The initial prescanning in order to get an overview of the infraclavicular anatomy, and adjust the ultrasound settings was not included in the procedure time. Measurement of procedure time was done using the stopwatch function of the MotionMonitor xGen software (Innovative Sports Training, Chicago, Illinois, USA).

The number of hand movements and the distance traveled by each hand were recorded using a Polhemus Patriot electromagnetic motion tracking system (Polhemus, Colchester, Vermont, USA) with the MotionMonitor xGen software as described in our previous studies. ${ }^{34}$ A cut-off velocity of $0.03 \mathrm{~mm} / \mathrm{s}$ and Butterworth cut-off frequency of $2.0 \mathrm{~Hz}$ were used to distinguish consecutive hand movements. Polhemus Micro Sensors 1.8 (Polhemus) was placed on the distal phalanx of the third finger on each hand of the anesthesiologists performing the block.

Observation of neuromuscular responses and paresthesia (defined as unpleasant sensations with distal radiation not synchronous with the pulse of the nerve stimulator) during the nerve block procedure was registered. Backflow of blood, and all adverse events were registered.

The numbers of injections were counted.

Tripartite test scores were used for sensory and motor block assessment. ${ }^{7}$ Sensory blocks were assessed by cold test, repeatedly touching the skin with an ice bag on predefined marked positions in the sensory area of the nerves (musculocutaneous, radial, median, ulnar and antebrachial cutaneous nerve). We used a sensory test scale where $0=$ normal sensation (volunteers could feel touch and coldness), 1=analgesia (volunteers could feel touch but not cold) and $2=$ anesthesia (no feeling at all). A first sensory assessment was done prior to the block procedure, and then every 5 th minute from $5 \mathrm{~min}$ to $30 \mathrm{~min}$ after the local anesthetics had been injected, and every 30th minute from $45 \mathrm{~min}$ to 240 min. Block assessment ended when all nerves involved in the (sensory) block had returned to 0 (normal sensation) or after $240 \mathrm{~min}$. Motor block was assessed prior to the block procedure and $30 \mathrm{~min}$ after local anesthetic injection was completed. The motor testing included the musculocutaneous, radial, median, and ulnar nerve. The following test score was used: $0=$ normal muscle strength, $1=$ reduced muscle strength, and $2=$ no muscle strength (paralysis).

Secondary outcomes derived by the sensory and motor test scores were block success rate, block onset time, and block duration. A block was defined as successful, when all five nerves distal to the elbow (musculocutaneous, radial, median, ulnar, and antebrachial cutaneous nerve) had a sensory test score of 1 or 2 (the volunteer did not feel coldness or they had no feeling at all) within $30 \mathrm{~min}$ after injection of local anesthetic. Motor block success included motor test scores of 1 or 2 (the volunteer had reduced muscle strength or they had no muscle strength at all) for all four nerves with muscle strength testing. Onset time was defined as the time between the end of local anesthetic injection and development of a successful sensory block. Adjusted onset time was set to $60 \mathrm{~min}$ if sensory block was not observed within the first hour of block assessment. Block duration was defined as the time from the end of local anesthetic injection until at least one of the nerves involved in the (sensory) block had a sensory test score of 0 (feeling of touch and coldness). The time to total block regression was defined by the time it took until all five nerves distal to the elbow had test scores of 0 .

Immediately after the block, the participants were asked about their experience of discomfort during the block procedure using a Numeric Rating Scale $(0=$ no discomfort, $10=$ worst discomfort imaginable). After finishing the block procedure, the anesthesiologists were asked about their confidence in block success ("How much confidence do you have in your block on a scale from 0 to 10 , where 0 is totally unlikely that the block will be successful, and 10 means that the block undoubtedly will be successful?").

\section{Statistical analysis}

Statistical analysis was done using Stata/SE V.15.1 (StataCorp LLC). The predefined main outcome variable in this study with an $\mathrm{AB} / \mathrm{BA}$ cross-over study design was procedure time. The null hypothesis that the difference between procedural times is zero was tested with the matched-paired t-test. Based on previous studies and pilot measurements, a SD of $40 \%$ could be expected for the procedure time. We considered a procedure time reduction of $25 \%$ as clinically significant. A sample size of 23 volunteers would have had $80 \%$ power to detect a difference in means of $25 \%$ assuming average time consumptions of 240 s (mean 1) and $180 \mathrm{~s}$ (mean 2) with a common SD of $96 \mathrm{~s}$ (with 0.5 covariance), using a one-sample t-test with $\alpha 0.05$ and $\beta 0.8$. To allow for missing data or dropouts, 26 volunteers were included. The binary outcomes (motor response, block success, paresthesia, backflow of blood) were analyzed with the McNemar asymptotic test, which is a test for the null hypothesis of equal event probabilities in the two periods and a matched-paired t-test was used for continuous data. Group allocation blinding was only broken on completion of the statistical analysis.

\section{RESULTS}

Twenty-six healthy volunteers gave their written informed consent and were included in the study (figure 3 ). There were no dropouts. The demographic data and morphological characteristics of the volunteers are presented in table 1 . 


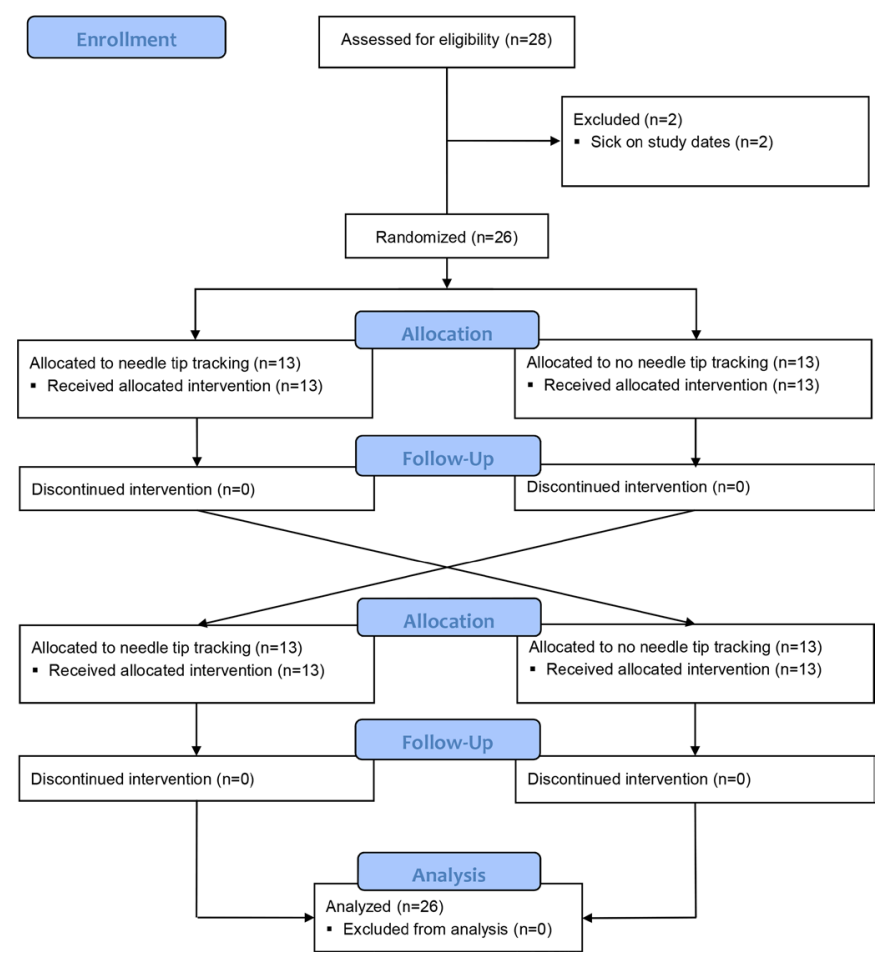

Figure 3 Modified Consolidated Standards of Reporting Trials flow diagram of study participants.

\section{Block performance}

Needle tip tracking did not statistically significantly reduce procedure time (primary outcome), needle path length or numbers of intended hand movements (secondary outcomes; table 2).

The mean number of injectates applied during the infraclavicular block procedure was similar with $(3.8 \pm 1.2)$ or without needle tip tracking $(3.8 \pm 1.1$; mean cross-over difference $(95 \% \mathrm{CI})=0.0(-0.7$ to 0.7$) ; \mathrm{p}=1.0)$. No statistically significant differences were found for paresthesia during the block procedures $(6 / 26$ (23\%) with needle tip tracking; 10/26 $(38 \%) ; \mathrm{p}=0.21)$, or the observation of muscle twitches with a constant stimulation current of $0.5 \mathrm{~mA} \mathrm{(22/26}$ (85\%) with needle tip tracking; 24/26 (92\%) without needle tip tracking; $\mathrm{p}=0.41$ ). Current thresholds to obtain a motor response were $0.81 \pm 0.27 \mathrm{~mA}$ with and $0.81 \pm 0.30 \mathrm{~mA}$ without needle tip tracking (mean cross-over difference $(95 \% \mathrm{CI})=0.00(-0.23$ to $0.23) \mathrm{mA} ; \mathrm{p}=0.98)$.

The mean discomfort experienced by the volunteers during the block procedure was $2.2 \pm 0.9$ with and $2.4 \pm 1.0$ without needle tip tracking (mean cross-over difference $(95 \% \mathrm{CI})-0.2$ $(-0.6$ to 0.2$) ; \mathrm{p}=0.23)$.

\begin{tabular}{ll}
\hline Table 1 & \\
\hline Age, years & $26.7(8.4)(21-57)$ \\
\hline Gender, $\mathrm{n}(\%)$ & \\
$\quad$ Male & $8(30.8)$ \\
$\quad$ Female & $18(69.2)$ \\
Weight, $\mathrm{kg}$ & $71.1(9.0)(57-87)$ \\
\hline Height, $\mathrm{cm}$ & $173.3(6.4)(163-186)$ \\
\hline Body mass index, $\mathrm{kg} / \mathrm{m}^{2}$ & $23.7(2.9)(19.1-31.6)$ \\
\hline
\end{tabular}

Continuous data are presented as mean (SD) (range) and categorical data are presented as frequency (percentage).

\section{Block characteristics}

Block success after 30 min was similar with (21/26 (81\%)) and without needle tip tracking $(18 / 26(69 \% ; \mathrm{p}=0.26))$. Motor block success with needle tip tracking $(8 / 26(31 \%))$ and without needle tip tracking (12/26 (46\%)) did not differ significantly. There was no significant differences in onset time (needle tip tracking, 23.6 $\pm 13.2 \mathrm{~min}$; without needle tip tracking, $27.0 \pm 21.0 \mathrm{~min}$; mean cross-over difference $(95 \% \mathrm{CI})=-3.4 \quad(-14.5$ to 7.7) min; $\mathrm{p}=0.53$ ), adjusted onset time (needle tip tracking $27.7 \pm 17.0 \mathrm{~min}$; without needle tip tracking, $31.0 \pm 20.3 \mathrm{~min}$; mean cross-over difference $(95 \% \mathrm{CI})=-3.3(-12.1$ to 5.6$) \mathrm{min}$; $\mathrm{p}=0.45$ ), block duration (needle tip tracking, $128.2 \pm 39.5 \mathrm{~min}$; without needle tip tracking, $143.2 \pm 48.0 \mathrm{~min}$; mean cross-over difference $(95 \% \mathrm{CI})=-15.0(-36.2$ to 6.29$) \mathrm{min} ; \mathrm{p}=0.16)$, and block release time (needle tip tracking, $212.3 \pm 52.9 \mathrm{~min}$; without needle tip tracking, $218.1 \pm 54.0 \mathrm{~min}$; mean cross-over difference $(95 \% \mathrm{CI})=-5.8(-21.8$ to 10.3$) \mathrm{min} ; \mathrm{p}=0.46)$. Confidence in block success was similar with needle tip tracking $(9.1 \pm 1.2)$ and without needle tip tracking $(8.9 \pm 1.0$; mean cross-over difference $(95 \% \mathrm{CI})=0.2(-0.5$ to 0.9$) ; \mathrm{p}=0.58)$.

\section{Period effect}

A non-significant period difference was observed for the main outcome variable: procedure time was $204.6 \pm 55.3 \mathrm{~s}$ during the first needle procedure and $185.2 \pm 57.8 \mathrm{~s}$ during the second procedure (mean period difference $=19.4 \mathrm{~s} ; 95 \% \mathrm{CI}-14.7$ to $53.5 \mathrm{~s}, \mathrm{p}=0.25)$.

\section{Adverse events}

Two volunteers experienced Horner syndrome during their first study day, both without needle tip tracking. There were no other adverse events.

\section{DISCUSSION}

In this randomized, controlled, observer blinded human volunteer trial, we compared in-plane ultrasound-guided infraclavicular blocks with and without the use of the needle tip tracking technology. A reduction of $12 \%$ in the procedure time, the main outcome variable in the study, was statistically not significant. For the secondary outcome measures, no statistically significant differences were found.

In a previous phantom study using porcine muscle tissue model, we found significantly reduced performance times and a significantly lower number of hand movements when the needle tip tracking technology was used for out-of-plane procedures. ${ }^{3}$ In contrast, differences for in-plane procedures were not significant. Hence, the results of our present study using an in-plane needle approach on infraclavicular blocks are in accordance with the findings of the phantom study.

Our recently published volunteer study showed significantly reduced numbers of hand movements and shorter path lengths measured by hand motion analysis when the needle tip tracking technology was used for out-of-plane lumbar plexus blocks. ${ }^{4}$ Visualizing a needle that is advanced transversally to the ultrasound plane in out-of-plane procedures is more difficult compared with in-plane procedures since only a portion of the needle can be seen in cross section. ${ }^{8}$ Incidence of unintended needle-to-nerve contacts is shown to be more frequent with outof-plane approaches. ${ }^{9}$

The use of a tracking technology might therefor be more helpful when used with out-of-plane procedures. However, failure to visualize the needle during in-plane procedures is a common error of less experienced anesthetists. ${ }^{10}$ Yet, the 


\begin{tabular}{llllll}
\hline & With NTT mean \pm SD & Without NTT mean \pm SD & $\begin{array}{l}\text { Cross-over difference } \\
\text { mean (95\% Cl) }\end{array}$ & P value & N \\
\hline Procedure time $(\mathrm{s})$ & $183.0 \pm 56.1$ & $206.8 \pm 56.2$ & $-23.8(-57.4$ to 9.8$)$ & 0.16 & 26 \\
Needle hand-movements $(\mathrm{n})$ & $55.7 \pm 23.6$ & $58.7 \pm 20.8$ & $-3.0(-13.2$ to 7.2$)$ & 0.55 & 26 \\
Needle hand-path length $(\mathrm{m})$ & $4.07 \pm 1.57$ & $4.15 \pm 1.08$ & $-0.08(-0.78$ to 0.63$)$ & 0.82 & 26 \\
Probe hand-movements $(\mathrm{n})$ & $1.1 \pm 2.4$ & $3.2 \pm 6.7$ & $-2.1(-5.2$ to 0.9$)$ & 0.16 & 23 \\
Probe hand-path length $(\mathrm{m})$ & $0.39 \pm 0.22$ & $0.79 \pm 1.05$ & $-0.40(-0.88$ to 0.08$)$ & 0.10 & 23 \\
\hline
\end{tabular}

NTT, needle tip tracking.

anesthesiologists involved in our study have been performing and teaching ultrasound-guided procedures on a regular basis during the last 10 years. Expert operators have been defined by having higher subspecialty training in regional anesthesia; they should also teach ultrasound-guided regional anesthesia and perform peripheral nerve blocks regularly. ${ }^{11}$ When assessing axillary blocks in their study, novices had three times more procedural errors compared with colleagues who met the expert criteria. Barrington et al demonstrated that trainees would require approximately 28 supervised trials with feedback before achieving competency in ultrasound needle visualization. ${ }^{12}$ The use of needle tracking technologies might give most benefits for less experienced operators and could be particularly helpful as an education tool. McLeod et al showed that the Onvision tracking technology can improve the performance of unexperienced anesthesiologists when conducting in-plane sciatic blocks in a cadaver study. ${ }^{13}$ Accordingly, McVicar et al showed a significant improvement of technical needling skills of novice trainees in an ex vivo model when using an electromagnetic needle tracking system. ${ }^{14}$ Since our present study was performed in healthy volunteers, it was considered as ethically questionable and inappropriate to involve less experienced colleagues.

Different needle tracking technologies have been developed in order to improve needle visualization, including electromagnetic tracking systems, fiber optic hydrophones and camera tracking. ${ }^{2}$ As far as we know, there are no randomized controlled trials for regional anesthesia techniques on human subjects investigating the effect of these methods. Randomized trials have only been performed in phantoms and cadavers. ${ }^{214-18}$

Many phantom models have successfully been used to simulate ultrasound-guided regional anesthesia in the past. ${ }^{19}$ While tofu, gelatin or muscle tissue phantoms provide acceptable feel for tissue and ultrasound attenuation, cadavers are considered as anatomically most relevant. ${ }^{13}$ However, block success, failure rates, or adverse events must be estimated indirectly in these models. $^{3}$

As a first in human testing of the Onvision needle tip tracking technology, we considered a volunteer trial appropriate. Procedure time was measured as a main outcome parameter. This outcome variable has been considered an appropriate proxy marker for needle control in numerous studies. ${ }^{20-24}$ However, procedure time is only a surrogate measure for the degree of skill and ease in block performance. Other clinical measures such as success rate or block failure might be considered more relevant. Yet, block failure is usually related to blocks that do not provide adequate anesthesia for a planned surgical procedure. In volunteer study, block failure can only be defined by sensory testing. To demonstrate a statistically significant difference in the failure rates of $13 \%$ in our study, more than 100 volunteers had to be included in a cross-over setup. ${ }^{6}$ The impact of needle tip tracking on complication rates can be seen as even more important. But, in order to show a difference in complication rates in a peripheral nerve block that is considered as relatively safe with few complications, an extremely large sample size would be needed. ${ }^{22}$

In our present volunteer trial, as well as in our previous studies on the needle tip tracking technology, procedure time was defined as the main outcome measure. ${ }^{34}$ The mean procedure time was $183 \mathrm{~s}$ with and $206.8 \mathrm{~s}$ without needle tip tracking. These time measurements are similar or slightly lower compared with the results from previous studies on ultrasound-guided infraclavicular blocks. This might be an indicator for the solid experience levels of the anesthesiologists performing the blocks in our study. ${ }^{25}$

Needle path lengths and number of hand movements measured by hand motion analysis have been used as a secondary outcome measure in our two previously published studies on needle tip tracking. ${ }^{3-5}$ Hand motion analysis permits a precise evaluation of dexterity and learning curves during surgical procedures and medical interventions. ${ }^{192627}$ For regional anesthesia, hand motion analysis has been validated in two previous studies involving supraclavicular plexus block and epidural insertion. ${ }^{27} 28$ In our previous studies, shorter path lengths and reduced numbers of hand movements indicated good dexterity and precise needle control during ultrasound-guided in-plane procedures aided by needle tip tracking. ${ }^{34}$

In the present study, we had an $81 \%$ block success rate with needle tip tracking and 69\% without needle tip tracking. The main reason for lower success rates compared with other studies on infraclavicular blocks is the low volume of $20 \mathrm{~mL} 20 \mathrm{mg} /$ $\mathrm{mL}$ lidocaine with $5 \mu \mathrm{g} / \mathrm{mL}$ epinephrine. ${ }^{29}$ We decided to use a low local anesthetic volume to ensure a short and predictable recovery. In a dose finding study, Tran et al determined a minimum effective volume of $35 \mathrm{~mL}$ for ultrasound-guided infraclavicular block. ${ }^{50}$ Since the volunteers where not supposed to undergo surgery, we did not depend on a complete block success.

Our study has several limitations. Most importantly, the operators performing the block were consultant anesthesiologists who are working with orthopedic anesthesia on a regular basis. Future studies might therefore investigate the effect of needle tip tracking used by unexperienced operators in a clinical setting.

Further, the healthy volunteers participating in our study differed from the standard patient population in our clinical practice. More obese or muscular patients, as well as elderly patients, can have a more challenging sonoanatomy and could probably benefit more from a needle tip tracking technology.

In the group of volunteers, there was a significant gender inequality (18 women and 8 men). Anatomically differences gender might potentially affect the results of a study on infraclavicular block. Due to the cross-over setup, both methods were tested in each of the participants. Hence, gender inequality should not have affected the results of our study. 
To ensure blinding of the observers, we used the same needles for all procedures both with active and inactive needle tip tracking. Yet, the tracking needles do not have a customized echogenic texture. The use of echogenic needles could have improved the visibility in the control procedures. However, based on pilot tests and our experience from our previous studies, we anticipate that any difference would be minimal.

The limited experience of the anesthesiologists with the needle tip tracking technology must also be taken into consideration. Since the Onvision needles obtained European Conformity (CE) marking just before the study start, the anesthesiologists were not able to use the needle tip tracking technology in clinical practice. They could only test the system for approximately 1 week in phantoms and Thiel cadavers.

Clinical studies in patients should be performed to verify or revise the results from our volunteer study.

\section{CONCLUSION}

When performing in-plane infraclavicular plexus block, the use of the needle tip tracking technology did not show a significant reduction in procedure time and did not significantly affect other markers of needle control.

Contributors TK, LR, LAR, MWF, PK and ARS planned and designed the study. TK and INO were the anesthesiologist who performed the blocks on the volunteers. LR and $\mathrm{AH}$ did the testing of the volunteers. ARS oversaw the study and was responsible for the envelopes with the randomization. TK, LR, AH, INO and ARS collected and screened the data. MWF performed statistical analysis and calculations. TK, $L R$, LAR, MWF, INO, PK and ARS interpreted the data. All authors wrote and edited the manuscript. ARS designed the figures.

Funding This project has received funding from the European Union's Horizon 2020 research and innovation programs (grant agreement 691262).

Competing interests B. Braun Melsungen AG and Philips Medical Systems International B.V. have been partners in the European Union's Horizon 2020 program. The main task for Oslo University Hospital was to conduct preclinical and clinical studies to evaluate the OnvisionR needle tip tracking technology. Ultrasound system and disposables used in the study were provided by B. Braun Melsungen AG and Philips Medical Systems International B.V.

Patient consent for publication Not required.

Ethics approval Ethical approval was provided by the Committee for Medical Research Ethics, Region South East, Oslo, Norway on August 8, 2018 (2018/1098/ REK-sør-øst). The study complied with the declaration of Helsinki.

Provenance and peer review Not commissioned; externally peer reviewed.

Data availability statement Data are available upon reasonable request. Please contact the corresponding author.

Open access This is an open access article distributed in accordance with the Creative Commons Attribution Non Commercial (CC BY-NC 4.0) license, which permits others to distribute, remix, adapt, build upon this work non-commercially, and license their derivative works on different terms, provided the original work is properly cited, an indication of whether changes were made, and the use is noncommercial. See: http://creativecommons.org/licenses/by-nc/4.0/.

\section{ORCID iD}

Trine Kåsine http://orcid.org/0000-0003-0868-9861

\section{REFERENCES}

1 Abrahams MS, Aziz MF, Fu RF, et al. Ultrasound guidance compared with electrical neurostimulation for peripheral nerve block: a systematic review and meta-analysis of randomized controlled trials. Br J Anaesth 2009;102:408-17.

2 Scholten HJ, Pourtaherian A, Mihajlovic N, et al. Improving needle tip identification during ultrasound-guided procedures in anaesthetic practice. Anaesthesia 2017;72:889-904.

3 Kåsine T, Romundstad L, Rosseland LA, et al. Needle tip tracking for ultrasoundguided peripheral nerve block procedures-An observer blinded, randomised, controlled, crossover study on a phantom model. Acta Anaesthesiol Scand 2019;63:1055-62.

4 Kåsine T, Romundstad L, Rosseland LA, et al. The effect of needle tip tracking on procedural time of ultrasound-guided lumbar plexus block: a randomised controlled trial. Anaesthesia 2020;75:72-9.

5 Sauter AR, Dodgson MS, Stubhaug A, et al. Electrical nerve stimulation or ultrasound guidance for lateral sagittal infraclavicular blocks: a randomized, controlled, observerblinded, comparative study. Anesth Analg 2008;106:1910-5.

6 Meinert CL. ClinicalTrials: design, conduct and analysis. Oxford University Press, 1986: 97-9.

7 Bendtsen TF, Nielsen TD, Rohde CV, et al. Ultrasound guidance improves a continuous popliteal sciatic nerve block when compared with nerve stimulation. Reg Anesth Pain Med 2011;36:181-4.

8 Sites BD, Brull R, Chan VWS, et al. Artifacts and pitfall errors associated with ultrasound-guided regional anesthesia. Part II: a pictorial approach to understanding and avoidance. Reg Anesth Pain Med 2007;32:419-33.

9 Ruiz A, Sala-Blanch X, Martinez-Ocón J, et al. Incidence of intraneural needle insertion in ultrasound-guided femoral nerve block: a comparison between the out-of-plane versus the in-plane approaches. Rev Esp Anestesiol Reanim 2014;61:73-7.

10 Sites BD, Spence BC, Gallagher JD, et al. Characterizing novice behavior associated with learning ultrasound-guided peripheral regional anesthesia. Reg Anesth Pain Med 2007;32:107-15

11 Ahmed OMA, O'Donnell BD, Gallagher AG, et al. Construct validity of a novel assessment tool for ultrasound-guided axillary brachial plexus block. Anaesthesia 2016;71:1324-31.

12 Barrington MJ, Wong DM, Slater B, et al. Ultrasound-Guided regional anesthesia: how much practice do novices require before achieving competency in ultrasound needle visualization using a cadaver model. Reg Anesth Pain Med 2012;37:334-9.

13 McLeod GA, McKendrick M, Taylor A, et al. An initial evaluation of the effect of a novel regional block needle with tip-tracking technology on the novice performance of cadaveric ultrasound-guided sciatic nerve block. Anaesthesia 2020;75:80-8.

14 McVicar J, Niazi AU, Murgatroyd H, et al. Novice performance of ultrasoundguided needling skills: effect of a needle guidance system. Reg Anesth Pain Med 2015:40:150-3.

15 Umbarje K, Tang R, Randhawa R, et al. Out-of-plane brachial plexus block with a novel SonixGPS(TM) needle tracking system. Anaesthesia 2013:68:433-4.

16 Beigi P, Rohling R, Salcudean T, et al. Needle trajectory and tip localization in real-time 3-D ultrasound using a moving Stylus. Ultrasound Med Biol 2015:41:2057-70.

17 Najafi M, Abolmaesumi P, Rohling R. Single-Camera Closed-Form real-time needle tracking for ultrasound-guided needle insertion. Ultrasound Med Biol 2015;41:2663-76

18 Xia W, Mari JM, West SJ, et al. In-Plane ultrasonic needle tracking using a fiber-optic hydrophone. Med Phys 2015;42:5983-91.

19 Farjad Sultan S, Shorten G, lohom G. Simulators for training in ultrasound guided procedures. Med Ultrason 2013;15:125-31.

20 Johnston DF, Stafford M. Dominant hand operating probe vs needle: a comparison study of ultrasound-guided needle placement in phantom models. Anaesthesia 2015;70:969-74.

21 Hewson DW, Bedforth NM, Hardman JG. Peripheral nerve injury arising in anaesthesia practice. Anaesthesia 2018:73:51-60.

22 Marhofer P, Fritsch G. Safe performance of peripheral regional anaesthesia: the significance of ultrasound guidance. Anaesthesia 2017;72:431-4.

23 Koscielniak-Nielsen ZJ, Stens-Pedersen HL, Lippert FK. Readiness for surgery after axillary block: single or multiple injection techniques. Eur J Anaesthesiol 1997:14:164-71.

24 Strid JMC, Sauter AR, Ullensvang K, et al. Ultrasound-Guided lumbar plexus block in volunteers; a randomized controlled trial. Br J Anaesth 2017:118:430-8.

25 Dhir S, Brown B, Mack P, et al. Infraclavicular and supraclavicular approaches to brachial plexus for ambulatory elbow surgery: a randomized controlled observerblinded trial. J Clin Anesth 2018;48:67-72.

26 Bann SD, Khan MS, Darzi AW. Measurement of surgical dexterity using motion analysis of simple bench tasks. World J Surg 2003;27:390-4.

27 Chin KJ, Tse C, Chan V, et al. Hand motion analysis using the Imperial College surgical assessment device: validation of a novel and objective performance measure in ultrasound-guided peripheral nerve blockade. Reg Anesth Pain Med 2011;36:213-9.

28 Hayter MA, Friedman Z, Bould MD, et al. Validation of the Imperial College surgical assessment device (ICSAD) for labour epidural placement. Can J Anaesth 2009;56:419-26.

29 Gürkan Y, Acar S, Solak M, et al. Comparison of nerve stimulation vs. ultrasoundguided lateral sagittal infraclavicular block. Acta Anaesthesiol Scand 2008;52:851-5.

30 Tran de QH, Dugani S, Dyachenko A, et al. Minimum effective volume of lidocaine for ultrasound-guided infraclavicular block. Reg Anesth Pain Med 2010;36:190-4. 